\title{
An efficient technique for higher order fractional differential equation
}

\author{
Ayyaz Ali ${ }^{1 *}$, Muhammad Asad lqbal' ${ }^{1}$, Qazi Mahmood UL-Hassan², Jamshad Ahmad ${ }^{3}$ \\ and Syed Tauseef Mohyud-Din ${ }^{1}$
}

*Correspondence: ali.ayyaz@ yahoo.com

${ }^{1}$ Department of Mathematics, Faculty of Sciences, HITEC University, Taxila, Pakistan

Full list of author information is available at the end of the article

\begin{abstract}
In this study, we establish exact solutions of fractional Kawahara equation by using the idea of $\exp (-\varphi(\eta)$ )-expansion method. The results of different studies show that the method is very effective and can be used as an alternative for finding exact solutions of nonlinear evolution equations (NLEEs) in mathematical physics. The solitary wave solutions are expressed by the hyperbolic, trigonometric, exponential and rational functions. Graphical representations along with the numerical data reinforce the efficacy of the used procedure. The specified idea is very effective, expedient for fractional PDEs, and could be extended to other physical problems.
\end{abstract}

Keywords: Kawahara equation, Fractional calculus, The exp $(-\varphi(\eta)$ )-expansion method, Traveling wave solutions, Modified Riemann-Liouville derivative

\section{Background}

Most of the scientific problems and phenomena arise nonlinearly in various fields of mathematical physics and applied sciences, such as fluid mechanics, plasma physics, optical fibers, solid-state physics, and geochemistry. The investigation of travelling wave solutions (Shawagfeh 2002; Ray and Bera 2005; Yildirim et al. 2011; Kilbas et al. 2006; He and Li 2010; Momani and Al-Khaled 2005; Odibat and Momani 2007; Abdou 2007; Nassar et al. 2011; Misirli and Gurefe 2011; Noor et al. 2008; Ozis and Koroglu 2008; Wu and He 2007; Yusufoglu 2008; Zhang 2007; Zhu 2007; Wang et al. 2008; Zayed et al. 2004; Sirendaoreji 2004; Ali 2011; Liang et al. 2011; He et al. 2012; Jawad et al. 2010; Zhou et al. 2003; Yıldırım and Kocak 2009; Elbeleze et al. 2013; Matinfar and Saeidy 2010; Ahmad 2014; Bongsoo 2009; Demiray and Pandir 2014, 2015; Lu 2012; Zayed and Amer 2014) of nonlinear evolution equations plays a significant role to look into the internal mechanism of nonlinear physical phenomena. Nonlinear fractional differential equations (FDEs) are a generalization of classical differential equations of integer order. The (FDEs) (Shawagfeh 2002; Ray and Bera 2005; Yildirim et al. 2011; Kilbas et al. 2006) have gained much importance due to exact interpretation of nonlinear phenomena. In recent years, considerable interest in fractional differential equations (He and Li 2010; Momani and Al-Khaled 2005; Odibat and Momani 2007) has been stimulated due to their numerous applications in different fields. However, many effective and powerful methods have been established and improved to study soliton solutions of nonlinear equations, such

(c) 2016 Ali et al. This article is distributed under the terms of the Creative Commons Attribution 4.0 International License (http:// creativecommons.org/licenses/by/4.0/, which permits unrestricted use, distribution, and reproduction in any medium, provided you give appropriate credit to the original author(s) and the source, provide a link to the Creative Commons license, and indicate if changes were made. 
as extended tanh-function method (Abdou 2007), tanh-function method (Nassar et al. 2011), Exp-function method (Misirli and Gurefe 2011; Noor et al. 2008; Ozis and Koroglu 2008; Wu and He 2007; Yusufoglu 2008; Zhang 2007; Zhu 2007), (G/G)-expansion method (Wang et al. 2008), homogeneous balance method (Zayed et al. 2004), auxiliary equation method (Sirendaoreji 2004), Jacobi elliptic function method (Ali 2011), Weierstrass elliptic function method (Liang et al. 2011), modified Exp-function method (He et al. 2012), modified simple equation method (Jawad et al. 2010), F-expansion method (Zhou et al. 2003), homotopy perturbation method (Yildırım and Kocak 2009), Fractional variational iteration method (Elbeleze et al. 2013), homotopy analysis method (Matinfar and Saeidy 2010), Reduced differential transform method (Ahmad 2014), Generalized Kudryashov method for time-fractional differential equations (Demiray and Pandir 2014), The first integral method for some time fractional differential equations( $\mathrm{Lu}$ 2012; Zayed and Amer 2014), New solitary wave solutions of Maccari system (Demiray and Pandir 2015), and so on.

In the present paper, we applied the exp $(-\varphi(\eta))$-expansion method to construct the appropriate solutions of fractional Kawahara equation and demonstrate the straightforwardness of the method. The fractional derivatives are used in modified Riemann-Liouville sense. The subject matter of this method is that the traveling wave solutions of nonlinear fractional differential equation can be expressed by a polynomial in $\exp (-\varphi(\eta))$.

$$
\left(\varphi^{\prime}(\eta)\right)=\exp (-\varphi(\eta))+\mu \exp (\varphi(\eta))+\lambda
$$

The article is organized as follows: In “Caputo's fractional derivative” section, the $\exp (-\varphi(\eta))$-expansion method is discussed. In "Description of exp $(-\varphi(\eta))$ expansion method" section, we exert the method to the nonlinear evolution equation pointed out above, in "Solution procedure" section, interpretation and graphical representation of results, and in "Graphical representation of the solutions" section conclusion and references are given.

\section{Caputo's fractional derivative}

In modelling physical phenomena, using differential equation of fractional order some drawbacks of Riemann-Liouville derivatives were observed In this section we set up the notations and recall some significant possessions.

Definition 1 A real function $f(x), x>0$ is said to be in space $C_{\alpha}, \alpha \in \Re$, if there exists a real number $p(>\alpha)$, such that

$$
f(x)=x^{p} f_{1}(x), \quad \text { where } f_{1}(x) \in C[0, \infty] .
$$

Definition 2 A real function $f(x), x>0$ is said to be in space $C_{\alpha}^{m}, m \in \mathbb{N} \cup\{0\}$, if $f^{(m)} \in C_{\alpha}$

Definition 3 Let $f \in C_{\alpha}$ and $\alpha \geq-1$, then the (left-sided) Riemann-Liouville integral of order $\mu, \mu>0$ is given by

$$
I_{t}^{\mu} f(x, t)=\frac{1}{\Gamma(\mu)} \int_{0}^{t}(t-\mathrm{T})^{\mu-1} f(x, \mathrm{~T}) d \mathrm{~T}, \quad t>0 .
$$


Definition 4 The (left sided) Caputo partial fractional derivative of $f$ with respect to $t$, $f \in C_{-1}^{m}, m \in \mathbb{N} \cup\{0\}$, is defined as:

$$
\begin{aligned}
D_{t}^{\mu} f(x, t) & =\frac{\partial^{m}}{\partial t^{m}} f(x, t), \mu=m \\
& =I_{t}^{m-\mu} \frac{\partial^{m}}{\partial t^{m}} f(x, t), \quad m-1 \leq \mu<m, m \in \mathbb{N}
\end{aligned}
$$

Note that

$$
\begin{aligned}
& I_{t}^{\mu} D_{t}^{\mu} f(x, t)=f(x, t)-\sum_{k=0}^{m-1} \frac{\partial^{k} f}{\partial t^{k}}(x, 0) \frac{t^{k}}{k !}, \quad m-1<\mu \leq m, m \in \mathbb{N} \\
& I_{t}^{\mu} t^{\nu}=\frac{\Gamma(v+1)}{\Gamma(\mu+v+1)} t^{\mu+v} .
\end{aligned}
$$

\section{Description of exp $(-\varphi(\eta))$ expansion method}

Now we explain the exp $(-\varphi(\eta))$-expansion method for finding traveling wave solutions of nonlinear evolution equations. Let us consider the general nonlinear FPDE of the type

$$
P\left(u, u_{t}, u_{x}, u_{x x}, u_{x x x}, \ldots, D_{t}^{\alpha} u, D_{x}^{\alpha} u, D_{x x}^{\alpha} u, \ldots\right)=0, \quad 0 \leq \alpha \leq 1,
$$

where $D_{t}^{\alpha} u, D_{x}^{\alpha} u, D_{x x}^{\alpha} u$ are the modified Riemann-Liouville derivatives of $u$ with respect to $t, x, x x$ respectively.

Using a transformation $\eta=k x+\frac{\omega t^{\alpha}}{\Gamma(1+\alpha)}+\eta_{0}, k, \omega, \eta_{0}$ are all constants with

$$
k, \omega \neq 0
$$

using the $\exp (-\varphi(\eta))$-expansion method we have to follow the following steps.

Step1. Combining the real variables $x$ and $t$ by a compound variable $\eta$ we assume

$$
u(x, t)=u(\eta)
$$

using the traveling wave variable Eqs. (10) and (8) is reduced to the following ODE for $u=u(\eta)$

$$
Q\left(u, u^{\prime}, u^{\prime \prime}, u^{\prime \prime \prime}, u, \ldots\right)=0,
$$

where $\mathrm{Q}$ is a function of $u(\eta)$ and its derivatives, prime denotes derivative with respect to $\eta$

Step2. Suppose the solution of Eq. (11) can be expressed by a polynomial in exp $(-\varphi(\eta))$ as follows

$$
u(\eta)=a_{n}(\exp (-\varphi(\eta)))^{n}+a_{n-1}(\exp (-\varphi(\eta)))^{n-1}+\cdots,
$$

where $a_{n}, a_{n-1}, \ldots$ and $\mathrm{V}$ are constants to determined later such that $a_{n} \neq 0$ and $\phi(\eta)$ satisfies equation Eq. (8)

Step3. By using the homogenous principal, we can evaluate the value of positive integer $n$ between the highest order linear terms and nonlinear terms of the highest order in 
Eq. (11). Our solutions now depend on the parameters involved in Eq. (1). So Eq. (1) provides the solutions from (13) to (16)

Case $1 \lambda^{2}-4 \mu>0$ and $\mu \neq 0$,

$$
\varphi(\eta)=\ln \left\{\frac{1}{2 \mu}\left(-\sqrt{\lambda^{2}-4 \mu} \tanh \left(\frac{\sqrt{\lambda^{2}-4 \mu}}{2}\left(\eta+c_{1}\right)\right)-\lambda\right)\right\},
$$

where $c_{1}$ is a constant of integration.

Case $2 \lambda^{2}-4 \mu<0$ and $\mu \neq 0$,

$$
\varphi(\eta)=\ln \left\{\frac{1}{2 \mu}\left(-\lambda+\sqrt{-\lambda^{2}+4 \mu} \tan \left(\frac{\sqrt{-\lambda^{2}+4 \mu}}{2}\left(\eta+c_{1}\right)\right)\right)\right\},
$$

Case $3 \mu=0$ and $\lambda \neq 0$,

$$
\varphi(\eta)=-\ln \left\{\frac{\lambda}{\exp \left(\lambda\left(\eta+c_{1}\right)\right)-1}\right\}
$$

Case $4 \lambda^{2}-4 \mu=0, \lambda \neq 0$, and $\mu \neq 0$,

$$
\varphi(\eta)=\ln \left\{\frac{2\left(\lambda\left(\eta+c_{1}\right)+2\right)}{\left(\lambda^{2}\left(\eta+c_{1}\right)\right)}\right\}
$$

Case $5 \lambda=0$, and $\mu=0$,

$$
\varphi(\xi)=\ln \left(\eta+c_{1}\right),
$$

Step4. Substitute Eq. (11) into Eq. (12) and using Eq. (1) the left hand side is converted into a polynomial in $\exp (-\varphi(\eta))$, equating each coefficient of this polynomial to zero, we obtain a set of algebraic equations for $a_{n}, \ldots \lambda, \mu$.

Step5. Eventually solving the algebraic system of equations obtained in step 4 by the use of Maple, we obtain the values of the constants $a_{n}, \ldots, \lambda$ and $\mu$. Substituting $a_{n}, \ldots$ and the general solution of Eq. (8) into solution Eq. (11), we obtain some valuable traveling wave solutions of Eq. (8).

\section{Solution procedure}

Consider the generalized form of fractional order nonlinear Kawahara equation.

$$
D_{t}^{\alpha} u+\beta u u_{x}+\alpha u_{x x x}-\delta u_{x x x x x}=0, \quad 0<\alpha \leq 1
$$

where $\alpha, \beta$ and $\delta$ are some nonzero parameters, taking $\alpha=1, \beta=1$ and $\delta=-1$, the model equation is given as. We can convert equation Eq. (18) into an ordinary differential equation.

$$
-V u^{\prime}+u u^{\prime}+u^{\prime \prime \prime}-u^{\prime \prime \prime \prime}=0,
$$

where the prime denotes the derivative with respect to $\eta$. Now integrating equation Eq. (19), we have, 


$$
-V u+\frac{1}{2} u^{2}+u^{\prime \prime}-u^{\prime \prime \prime \prime}+C=0
$$

Balancing the $u^{\prime \prime \prime \prime}$ and $u^{2}$ by using homogenous principal, we have

$$
\begin{aligned}
2 M & =M+4, \\
M & =4 .
\end{aligned}
$$

Then the trial solution of equation Eq. (19) can be expressed as follows,

$$
u(\eta)=a_{0}+a_{1}(\exp (-\varphi(\eta)))+a_{2}(\exp (-\varphi(\eta)))^{2}+a_{3}(\exp (-\varphi(\eta)))^{3}+a_{4}(\exp (-\varphi(\eta)))^{4}
$$

where $a_{4} \neq 0, a_{0}, a_{1}, a_{2}$ and $a_{3}$ are constants to determined, while $\lambda, \mu$ are arbitrary constants.

Substituting $u, u^{\prime}, u^{\prime \prime}, u^{\prime \prime \prime}, u^{\prime \prime \prime \prime}, u^{2}$ into Eq. (20) and then equating the coefficients of $\exp (-\varphi(\eta))$ to zero, we get the set of algebraic equations, we obtain the following solution.

\section{Solution 1}

$$
\left\{\begin{array}{l}
V=1680 \mu^{2}-\frac{36}{169}-a_{0}, a_{0}=a_{0}, a_{1}=-\frac{3360}{13} \mu^{2} \sqrt{676 \mu+13} \\
a_{2}=\frac{1680}{13}(1+78 \mu) \mu^{2}, \lambda=-\frac{1}{13} \sqrt{676 \mu+13}, \\
a_{3}=-\frac{3360}{13} \mu^{3} \sqrt{676 \mu+13}, a_{4}=1680 \mu^{4} \\
C=-\frac{60480}{169} \mu^{2}+1411200 \mu^{4}-1680 \mu^{2} a_{0}+\frac{36}{169} a_{0}+\frac{1}{2}+a_{0}^{2}
\end{array},\right.
$$

where $\lambda$ and $\mu$ are arbitrary constants.

Now substituting the values into Eq. (21), we obtain,

$$
\begin{aligned}
u(\eta)= & a_{0}+-\frac{3360}{13} \mu^{2} \sqrt{676 \mu+13}(\exp (-\varphi(\eta)))+\frac{1680}{13}(1+78 \mu) \mu^{2}(\exp (-\varphi(\eta)))^{2} \\
& -\frac{3360}{13} \mu^{3} \sqrt{676 \mu+13}(\exp (-\varphi(\eta)))^{3}+1680 \mu^{4}
\end{aligned}
$$

Now substituting Eq. (13), (14), (15), (16) and (17) into Eq. (23) respectively, we get the following five traveling wave solutions of the Kawahara equation.

Case 1 When $\lambda^{2}-4 \mu>0$ and $\mu \neq 0$, we obtain the hyperbolic function traveling wave solution.

$$
\begin{aligned}
u_{1}(\eta) & =a_{0}-1680 \mu^{2}+\frac{105}{169} \\
& -\frac{210}{169} \tanh \left(\frac{1}{4394}\left(169 x+\frac{283920 t^{\alpha} \mu^{2}}{\Gamma(\alpha+1)}-\frac{36 t^{\alpha}}{\Gamma(\alpha+1)}-\frac{169 t^{\alpha} a_{0}}{\Gamma(\alpha+1)}\right) \sqrt{13}\right)^{2} \\
& +\frac{105}{169} \tanh \left(\frac{1}{4394}\left(\begin{array}{c}
169 x+\frac{283920 t^{\alpha} \mu^{2}}{\Gamma(\alpha+1)} \\
-\frac{36 t^{\alpha}}{\Gamma(\alpha+1)}-\frac{169 t^{\alpha} a_{0}}{\Gamma(\alpha+1)}
\end{array}\right) \sqrt{13}\right)^{4}
\end{aligned}
$$


Case 2 When $\lambda^{2}-4 \mu<0$ and $\mu \neq 0$, we obtain trigonometric solution

$$
\begin{aligned}
& u_{2}(\eta)=a_{0}-1680 \mu^{2}+\frac{105}{169} \\
& -\frac{210}{169} \tanh \left(\frac{1}{4394}\left(169 x+\frac{283920 t^{\alpha} \mu^{2}}{\Gamma(\alpha+1)}-\frac{36 t^{\alpha}}{\Gamma(\alpha+1)}-\frac{169 t^{\alpha} a_{0}}{\Gamma(\alpha+1)}\right) \sqrt{13}\right)^{2} \\
& +\frac{105}{169} \tanh \left(\frac{1}{4394}\left(\begin{array}{c}
169 x \\
+\frac{283920 t^{\alpha} \mu^{2}}{\Gamma(\alpha+1)} \\
-\frac{36 t^{\alpha}}{\Gamma(\alpha+1)} \\
-\frac{169 t^{\alpha} a_{0}}{\Gamma(\alpha+1)}
\end{array}\right) \sqrt{13}\right)^{4}
\end{aligned}
$$

Case 3 When $\mu=0$ and $\lambda \neq 0$, we obtain exponential solution.

$$
\begin{aligned}
u_{3}(\eta)=1 /(52 \mu+1)^{2} & {\left[a_{0}+1135680 \mu^{4} e^{\frac{-1}{2197} \sqrt{676 \mu+13}\left(169 x+\frac{283920 t^{\alpha} \mu^{2}}{\Gamma(\alpha+1)}-\frac{36 t^{\alpha}}{\Gamma(\alpha+1)}-\frac{169 t^{\alpha} a_{0}}{\Gamma(\alpha+1)}\right)}\right.} \\
& +43680 \mu^{3} e^{\frac{-1}{2197} \sqrt{676 \mu+13}\left(169 x+\frac{283920 t^{\alpha} \mu^{2}}{\Gamma(\alpha+1)}-\frac{36 t^{\alpha}}{\Gamma(\alpha+1)}-\frac{169 t^{\alpha} a_{0}}{\Gamma(\alpha+1)}\right)} \\
& +1680 \mu^{2} e^{\frac{-2}{2197} \sqrt{676 \mu+13}\left(169 x+\frac{283920 t^{\alpha} \mu^{2}}{\Gamma(\alpha+1)}-\frac{36 t^{\alpha}}{\Gamma(\alpha+1)}-\frac{169 t^{\alpha} a_{0}}{\Gamma(\alpha+1)}\right)} \\
& +1703520 \mu^{4} e^{\frac{-2}{2197} \sqrt{676 \mu+13}\left(169 x+\frac{283920 t^{\alpha} \mu^{2}}{\Gamma(\alpha+1)}-\frac{36 t^{\alpha}}{\Gamma(\alpha+1)}-\frac{169 t^{\alpha} a_{0}}{\Gamma(\alpha+1)}\right)} \\
& +\cdots 87360 \mu^{3} e^{\frac{-2}{2197} \sqrt{676 \mu+13}\left(169 x+\frac{283920 t^{\alpha} \mu^{2}}{\Gamma(\alpha+1)}-\frac{36 t^{\alpha}}{\Gamma(\alpha+1)}-\frac{169 t^{\alpha} a_{0}}{\Gamma(\alpha+1)}\right)} \\
& \left.-4258800 \mu^{4}-174720 \mu^{3}\right],
\end{aligned}
$$

Case 4 When $\lambda^{2}-4 \mu=0, \lambda \neq 0$, and $\mu \neq 0$, we obtain rational function solution.

$$
u_{4}(\eta)=\frac{\left[\begin{array}{l}
-\frac{84464623357806182400\left(t^{\alpha}\right)^{3} \mu^{6}}{\Gamma(\alpha+1)^{3}} \\
+\frac{14434947944202240\left(t^{\alpha}\right)^{3} \mu^{4}}{\Gamma(\alpha+1)^{3}} \\
+\cdots-\frac{71262235786560 \sqrt{676 \mu+13} a_{0}^{2}\left(t^{\alpha}\right)^{2} \mu^{2}}{\Gamma(\alpha+1)^{2}}
\end{array}\right]}{\left[\sqrt{676 \mu+13}\left(\begin{array}{c}
169 x+\frac{283920 t^{\alpha} \mu^{2}}{\Gamma(\alpha+1)} \\
-\frac{36 t^{\alpha}}{\Gamma(\alpha+1)}-\frac{169 t^{\alpha} a_{0}}{\Gamma(\alpha+1)}
\end{array}\right)(52 \mu+1)^{4}\right]},
$$

Case 5 when $\lambda=0$, and $\mu=0$, we obtain rational function solution.

$u_{5}(\eta)=-\frac{20160 \mu^{3} x t^{\alpha} a_{0}}{\Gamma(\alpha+1)}-\frac{3360}{13} \frac{\mu^{2} x t^{\alpha} a_{0}}{\Gamma(\alpha+1)}-\frac{26127360}{28561} \frac{\left(t^{\alpha}\right)^{3} a_{0} \mu^{4} x}{\Gamma(\alpha+1)^{3}}+\cdots \frac{3360}{13} \mu^{2} \sqrt{676 \mu+13 x}$ 
Solution 2

$$
\left\{\begin{array}{l}
V=1680 \mu^{2}-\frac{36}{169}-a_{0}, a_{0}=a_{0}, a_{1}=\frac{3360}{13} \mu \sqrt{676 \mu+13}, \\
a_{2}=\frac{1680}{13}+10080 \mu, \lambda=\frac{1}{13} \sqrt{676 \mu+13} \\
a_{3}=\frac{3360}{13} \sqrt{676 \mu+13}, a_{4}=1680 \\
C=-\frac{60480}{169} \mu^{2}+1411200 \mu^{4}-1680 \mu^{2} a_{0}+\frac{36}{169} a_{0}+\frac{1}{2} a_{0}^{2}
\end{array},\right.
$$

\section{Solution 3}

$$
\left\{\begin{array}{l}
V=1680 \mu^{2}-\frac{36}{169}-a_{0}, a_{0}=a_{0}, a_{1}=-\frac{3360}{13} \mu \sqrt{676 \mu+13}, \\
a_{2}=\frac{1680}{13}+10080 \mu, \lambda=-\frac{1}{13} \sqrt{676 \mu+13}, \\
a_{3}=-\frac{3360}{13} \sqrt{676 \mu+13}, a_{4}=1680, \\
C=-\frac{60480}{169} \mu^{2}+1411200 \mu^{4}-1680 \mu^{2} a_{0}+\frac{36}{169} a_{0}+\frac{1}{2} a_{0}^{2}
\end{array},\right.
$$

Solution 4

$\left\{\begin{array}{l}V=560 \mu^{2}-\frac{280}{13} \mu-a_{0}+\frac{4371}{33800}-\frac{483}{33800} \iota \sqrt{31}+\frac{14}{845} \mu(-2015+67600 \mu+195 \iota \sqrt{31}), \ldots, \\ a_{0}=a_{0}, a_{4}=1680\end{array}\right.$

Similarly, we can find the other exact solution of remaining solutions, while one solution is analyzed.

\section{Graphical representation of the solutions}

The graphical illustrations of the solutions are given below in the figures with the aid of Maple.

\section{Physical interpretation}

The proposed method provides more general and abundant new solitary wave solutions with some free parameters. The traveling wave solutions have its extensive significance to interpret the inner structures of the natural phenomena. We have explained the different types of solitary wave solutions by setting the physical parameters as special values. In this paragraph, we will explain the physical elucidation of the solutions for the Kawahara equation for $\mathbf{a}_{0}=11.1, \mu=-0.0002, x=15, \alpha=0.50, u_{1}$ shows the singular solitary wave solution as shown in Figs. 1, 2, 3). Figure 4 shows the shape of the singular kink wave solution of $\mathbf{u}_{2}$ for $\mathbf{a}_{0}=5.1, \mu=0.002, x=2, \alpha=0.75$. Again singular Kink solution obtained in Fig. 5 of $\mathbf{u}_{2}$ for $\boldsymbol{a}_{\mathbf{0}}=\mathbf{5 . 1}, \mu=\mathbf{0 . 0 0 2}, \boldsymbol{x}=\mathbf{2}, \alpha=\mathbf{0 . 5 0}$ (Figs. $6,7,8,9,10,11$ ). Finally simple kink solution got from $\boldsymbol{u}_{\mathbf{5}}$ for the choice of $\mathbf{a}_{0}=-\mathbf{2}, \boldsymbol{\mu}=\mathbf{1 4}, \mathbf{x}=\mathbf{1 8}, \alpha=\mathbf{0 . 7 5}$. which is shown in Fig. 12. In one asymptotic state to another asymptotic state, kink solitons are upsurge or descent. Such solitons are called topological solitons. The other exact solutions could be obtained from the remaining solution sets. 


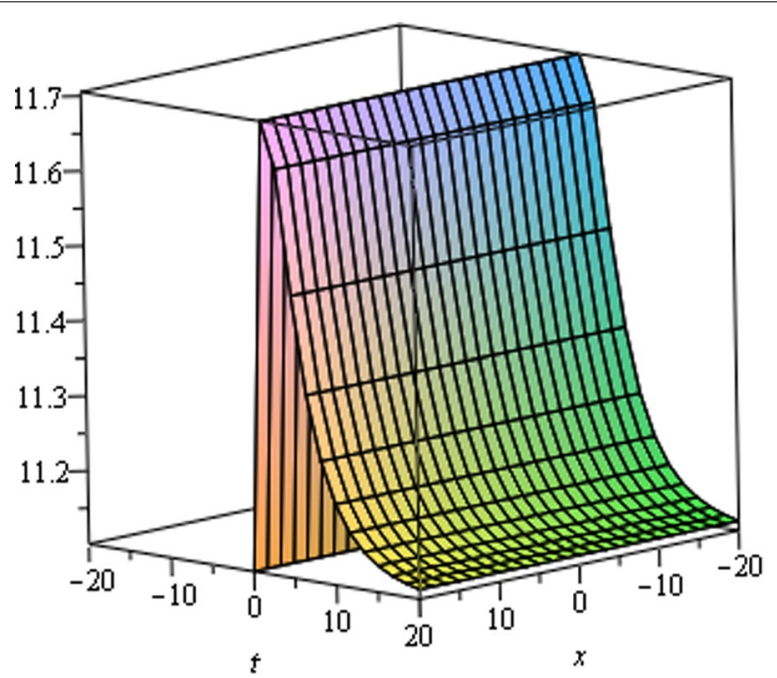

Fig. 1 Singular solitary wave solution $u_{1}(\eta)$ when $\mathrm{a}_{0}=11.1, \mu=-0.0002, \mathrm{x}=15, \alpha=0.25$

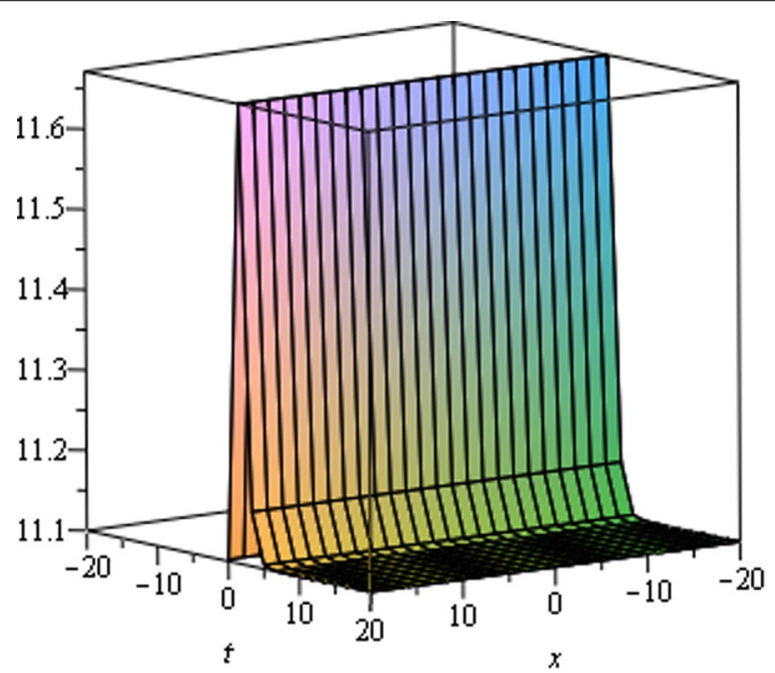

Fig. 2 Singular solitary wave solution $u_{1}(\eta)$ when $a_{0}=11.1, \mu=-0.0002, x=15, \alpha=0.50$.

\section{Numerical discussion}

We have obtained the exact solutions (29), (30) and (31) in the above study and to know the correctness we have matched those solutions with the exact solution (Bongsoo 2009).We note that the absolute errors given in the tables from the solutions we have obtained are very precise and accurate.

$$
u(x, t)=\frac{420 \beta}{164 \alpha \gamma}[n(x, t)]\left[\frac{n(x, t)}{2}+1\right]^{2}
$$




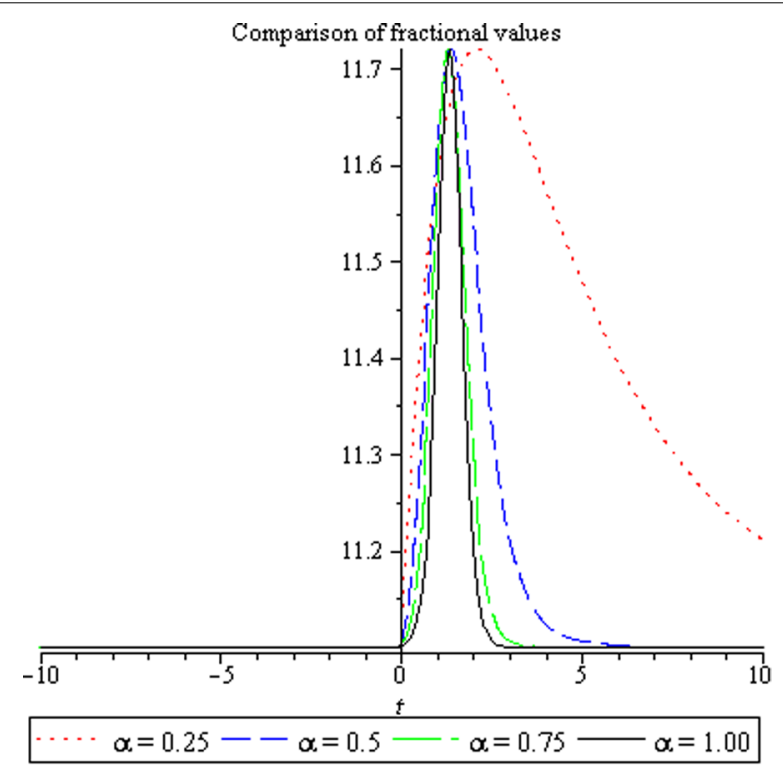

Fig. 3 Comparison of solutions for different values of $\alpha$

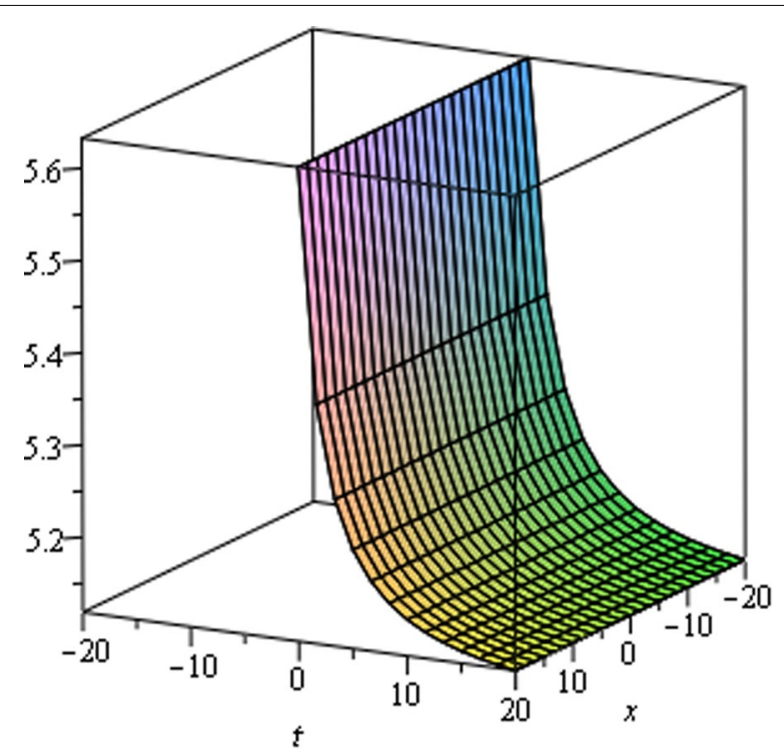

Fig. 4 Singular Kink wave solution $u_{2}(\eta)$ when $a_{0}=5.1, \mu=0.002, x=2, \alpha=0.50$

where

$$
n(x, t)=\frac{1-\left\{\cosh \left[\sqrt{\frac{\beta}{13 \gamma}}\left(x-\frac{36 \beta^{2} t}{169 \gamma}+m\right)\right]+\sqrt{p^{2}+1}\right\}}{\left\{\sinh \left[\sqrt{\frac{\beta}{13 \gamma}}\left(x-\frac{36 \beta^{2} t}{169 \gamma}+m\right)\right]+p\right\}}
$$




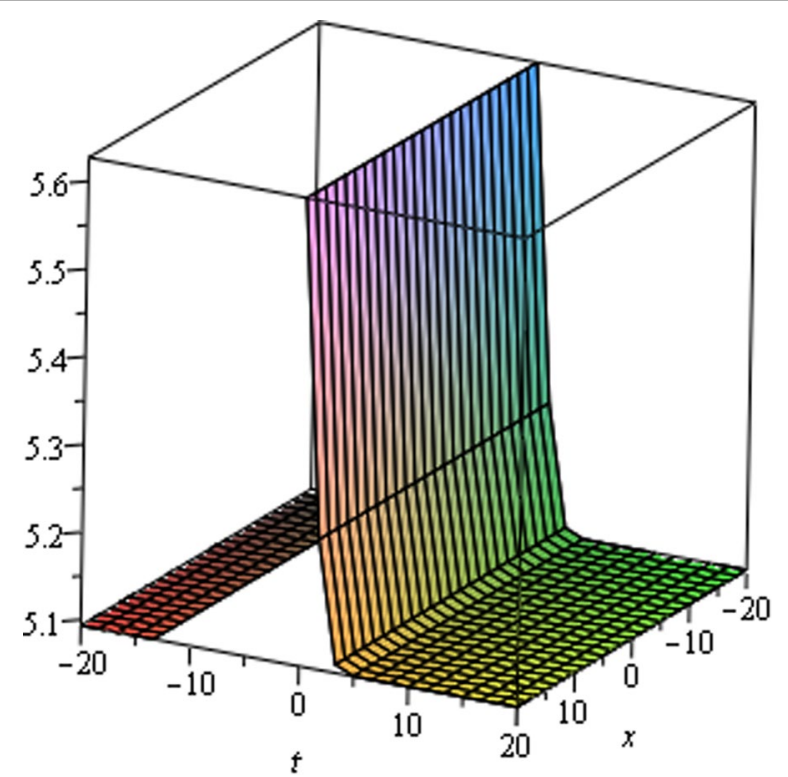

Fig. 5 Singular Kink wave solution $u_{2}(\eta)$ when $a_{0}=5.1, \mu=0.002, x=2, \alpha=0.75$

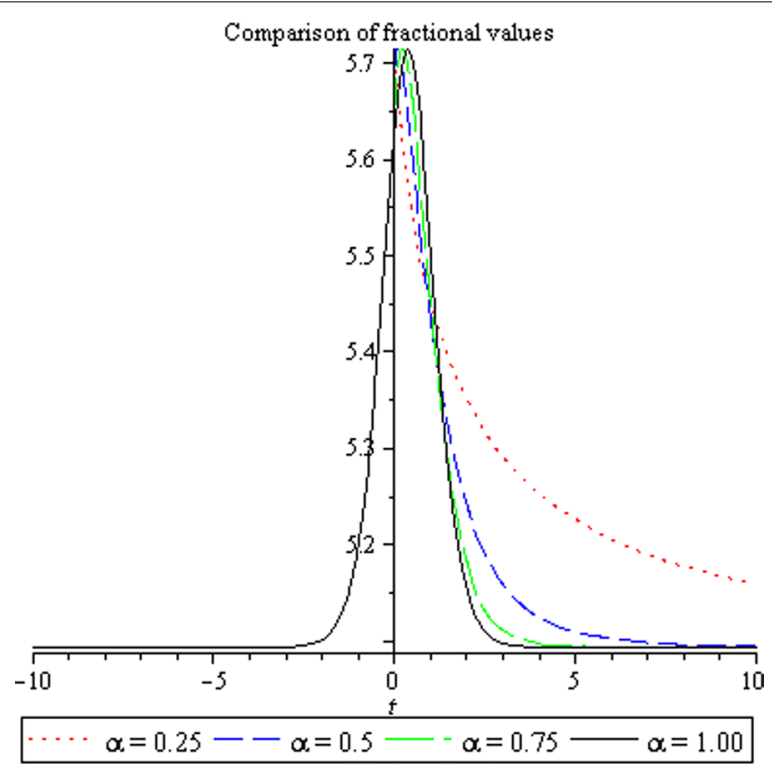

Fig. 6 Comparison of solutions for different values of $\alpha$

\section{Conclusion}

With the help of a suitable transformation and the exp $(-\varphi(\eta))$-expansion method, we obtained different types of exact solutions for fractional Kawahara equation. The obtained results show that the proposed technique is effective and capable for solving nonlinear fractional partial differential equations. In this research, some exact solitary wave solutions, mostly solitons and kinks solutions are obtained through the hyperbolic, 


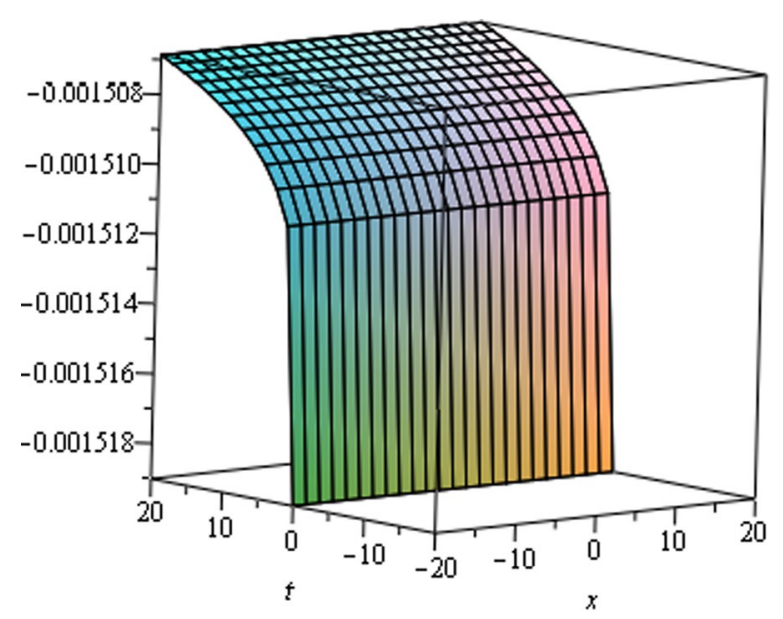

Fig. 7 Singular Kink wave solution $u_{3}(\eta)$ when $a_{0}=0.0001, \mu=0.001, x=6, \alpha=0.25$

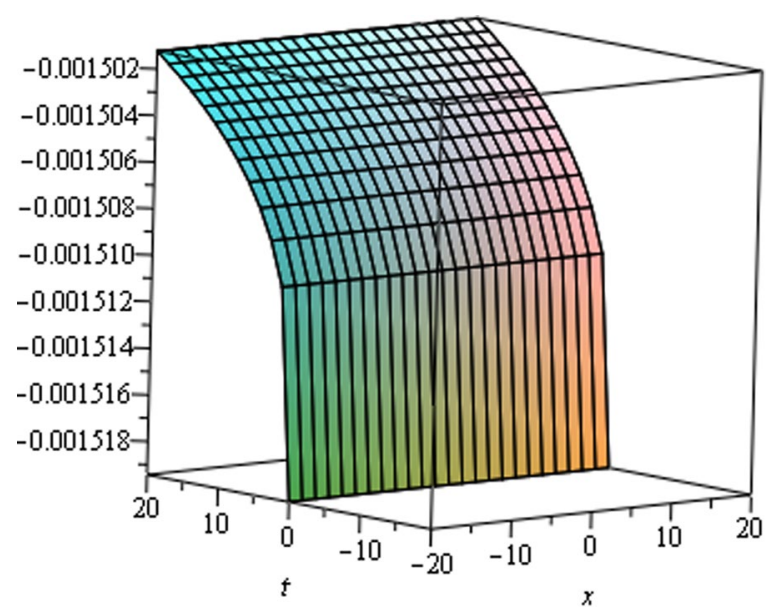

Fig. 8 Singular Kink wave solution $u_{3}(\eta)$ when $a_{0}=0.0001, \mu=0.001, x=6, \alpha=0.15$

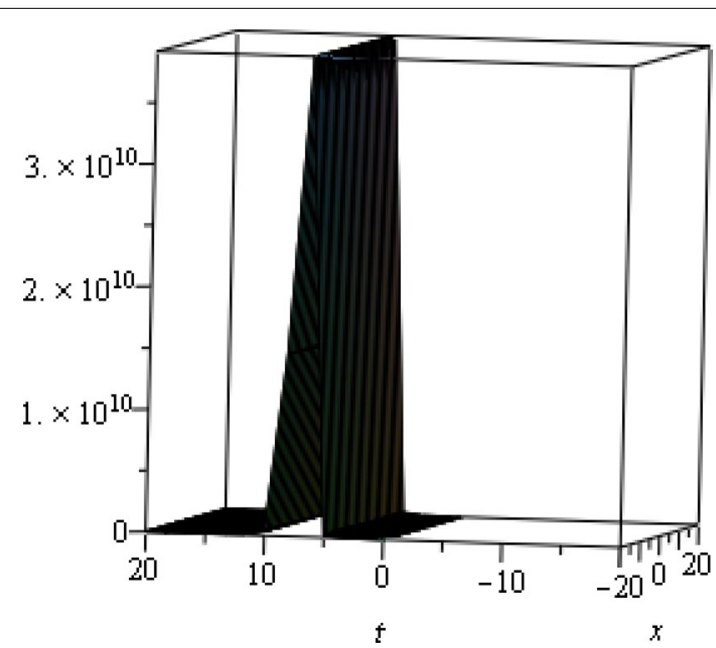

Fig. 9 Singular solitary wave solution $u_{4}$ when $a_{0}=0.01, \mu=0.001, x=0.1, \alpha=0.50$ 


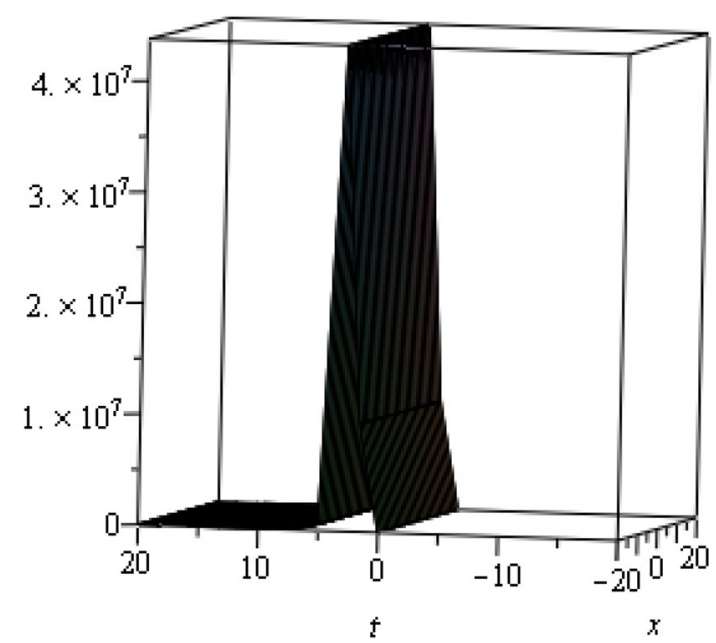

Fig. 10 Singular solitary wave solution $u_{4}$ when $a_{0}=0.01, \mu=0.001, x=0.1, \alpha=0.25$

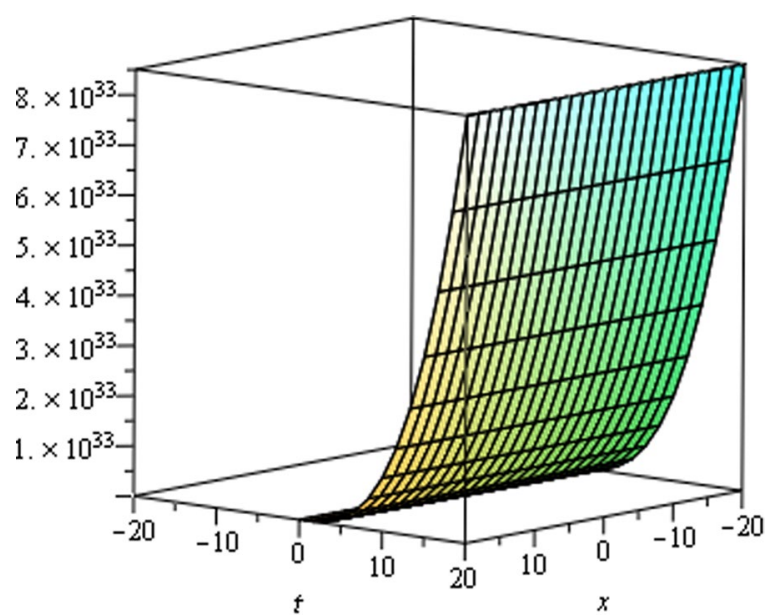

Fig. 11 Singular Kink wave solution $u_{5}(\eta)$ when $a_{0}=-2, \mu=14, x=18 \alpha=1.00$

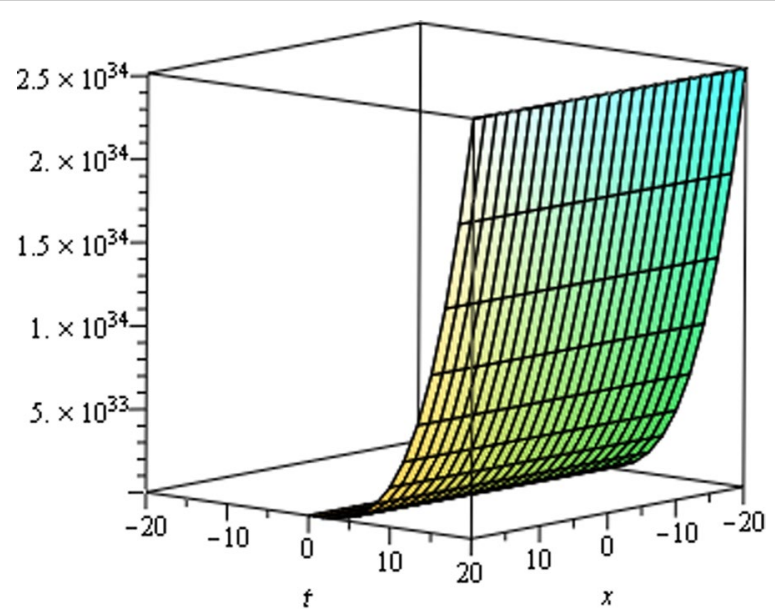

Fig. 12 Singular Kink wave solution $u_{5}(\eta)$ when $a_{0}=-2, \mu=14, x=18 \alpha=0.75$. 
Table 1 Comparison of the absolute errors for the exact solution obtained by (29) with the exact solution (32) when

\begin{tabular}{llllll}
\hline $\mathbf{x} \backslash \mathbf{t}$ & $\mathbf{0 . 5}$ & $\mathbf{1}$ & $\mathbf{1 . 5}$ & $\mathbf{2}$ & $\mathbf{2 . 5}$ \\
\hline 0.5 & $2.07927 \mathrm{E}-05$ & $2.16050 \mathrm{E}-05$ & $2.24665 \mathrm{E}-05$ & $2.33804 \mathrm{E}-05$ & $2.43496 \mathrm{E}-05$ \\
1.0 & $1.69635 \mathrm{E}-05$ & $1.75796 \mathrm{E}-05$ & $1.82331 \mathrm{E}-05$ & $1.89262 \mathrm{E}-05$ & $1.96615 \mathrm{E}-05$ \\
1.5 & $1.39182 \mathrm{E}-05$ & $1.43854 \mathrm{E}-05$ & $1.48811 \mathrm{E}-05$ & $1.54068 \mathrm{E}-05$ & $1.59644 \mathrm{E}-05$ \\
2.0 & $1.14683 \mathrm{E}-05$ & $1.18227 \mathrm{E}-05$ & $1.21985 \mathrm{E}-05$ & $1.25972 \mathrm{E}-05$ & $1.30201 \mathrm{E}-05$ \\
2.5 & $9.47090 \mathrm{E}-06$ & $9.73956 \mathrm{E}-06$ & $1.00245 \mathrm{E}-05$ & $1.03269 \mathrm{E}-05$ & $1.06475 \mathrm{E}-05$ \\
\hline
\end{tabular}

Table 2 Comparison of absolute errors obtained by (30) with the exact solution (32) when

\begin{tabular}{llllll}
\hline $\mathbf{x} \backslash \mathbf{t}$ & $\mathbf{0 . 5}$ & $\mathbf{1}$ & $\mathbf{1 . 5}$ & $\mathbf{2}$ & $\mathbf{2 . 5}$ \\
\hline 0.5 & $2.80883 \mathrm{E}-05$ & $2.69054 \mathrm{E}-05$ & $2.57900 \mathrm{E}-05$ & $2.47385 \mathrm{E}-05$ & $2.37470 \mathrm{E}-05$ \\
1.0 & $3.40754 \mathrm{E}-05$ & $3.25163 \mathrm{E}-05$ & $3.10463 \mathrm{E}-05$ & $2.96603 \mathrm{E}-05$ & $2.83534 \mathrm{E}-05$ \\
1.5 & $4.21575 \mathrm{E}-05$ & $4.01031 \mathrm{E}-05$ & $3.81659 \mathrm{E}-05$ & $3.63394 \mathrm{E}-05$ & $3.46171 \mathrm{E}-05$ \\
2.0 & $5.29970 \mathrm{E}-05$ & $5.02905 \mathrm{E}-05$ & $4.77384 \mathrm{E}-05$ & $4.53318 \mathrm{E}-05$ & $4.30626 \mathrm{E}-05$ \\
2.5 & $6.74652 \mathrm{E}-05$ & $6.39005 \mathrm{E}-05$ & $6.05389 \mathrm{E}-05$ & $5.73689 \mathrm{E}-05$ & $5.43796 \mathrm{E}-05$ \\
\hline
\end{tabular}

Table 3 Comparison of absolute errors obtained by (31) with the exact solution (32) when

\begin{tabular}{llllll}
\hline $\mathbf{x} \backslash \mathbf{t}$ & $\mathbf{0 . 5}$ & $\mathbf{1}$ & $\mathbf{1 . 5}$ & $\mathbf{2}$ & $\mathbf{2 . 5}$ \\
\hline 0.5 & $1.11802 \mathrm{E}-02$ & $1.10649 \mathrm{E}-02$ & $1.09508 \mathrm{E}-02$ & $1.08381 \mathrm{E}-02$ & $1.07266 \mathrm{E}-02$ \\
1.0 & $1.08009 \mathrm{E}-02$ & $1.06829 \mathrm{E}-02$ & $1.05663 \mathrm{E}-02$ & $1.04512 \mathrm{E}-02$ & $1.03374 \mathrm{E}-02$ \\
1.5 & $1.03542 \mathrm{E}-02$ & $1.02348 \mathrm{E}-02$ & $1.01170 \mathrm{E}-02$ & $1.00008 \mathrm{E}-02$ & $9.88610 \mathrm{E}-03$ \\
2.0 & $9.84771 \mathrm{E}-03$ & $9.72834 \mathrm{E}-03$ & $9.61069 \mathrm{E}-03$ & $9.49476 \mathrm{E}-03$ & $9.38053 \mathrm{E}-03$ \\
2.5 & $9.28994 \mathrm{E}-03$ & $9.17198 \mathrm{E}-03$ & $9.05588 \mathrm{E}-03$ & $8.94165 \mathrm{E}-03$ & $8.82927 \mathrm{E}-03$ \\
\hline
\end{tabular}

trigonometric, exponential and rational functions. It is observed that the proposed method fully validate the competence and reliability of computational work as evident from Tables 1, 2 and 3 and may be utilized for other physical problems.

\section{Authors' contributions}

The work was carried out in cooperation among all the authors (AA, STM, MAI, QMU and JA). All authors have a good involvement to plan the paper, and to execute the analysis of this research work together. All authors read and approved the final manuscript.

\section{Author details \\ ${ }^{1}$ Department of Mathematics, Faculty of Sciences, HITEC University, Taxila, Pakistan. ${ }^{2}$ Department of Mathematics, Fac- ulty of Basic Sciences, University of Wah, Wah Cantonment, Pakistan. ${ }^{3}$ Department of Mathematics, Faculty of Sciences,} University of Gujrat, Gujrat, Pakistan.

\section{Competing interests}

The authors declare that they have no competing interests.

Received: 2 October 2015 Accepted: 17 February 2016

Published online: 05 March 2016

References

Abdou MA (2007) The extended tanh-method and its applications for solving nonlinear physical models. Appl Math Comput 190:988-996 
Ahmad J, Mohyud-Din ST (2014) An efficient algorithm for some highly nonlinear fractional PDEs in mathematical physics. PLoS One 9(12):e109127. doi:10.1371/journal.pone.0109127

Ali AT (2011) New generalized Jacobi elliptic function rational expansion method. J Comput Appl Math 235:4117-4127 Bongsoo J (2009) New exact travelling wave solutions of Kawahara type equations. J Nonlinear Anal 70:510-515

Demiray ST, Pandir Y, Bulut H (2014). Generalized Kudryashov method for time-fractional differential equations, abstract and applied analysis, vol 2014

Demiray ST, Pandir Y, Bulut H (2015) New solitary wave solutions of Maccari system. Ocean Eng 15(103):153-159

Elbeleze AA, Kilicman A, Taib BM (2013) Fractional variational iteration method and its application to fractional partial differential equation. Math Probl Eng 2013, Article ID 543848. doi:10.1155/2013/543848

He JH, Li ZB (2010) Fractional complex transform for fractional differential equations. Comp Math Appl 15(5):970-973

He Y, Li S, Long Y (2012) Exact solutions of the Klein-Gordon equation by modified exp-function method. Int Math Forum 7(4):175-182

Jawad AJM, Petkovic MD, Biswas A (2010) Modified simple equation method for nonlinear evolution equations. Appl Math Comput 217:869-877

Kilbas AA, Srivastava HM, Trujillo JJ (2006) Theory and applications of fractional differential equations. Comput Math Appl 204:1269-1274

Liang MS et al (2011) A method to construct Weierstrass elliptic function solution for nonlinear equations. Int J Mod Phys B 25(4):1931-1939

Lu B (2012) The first integral method for some time fractional differential equations. J Math Anal Appl 395:684-693

Matinfar M, Saeidy M (2010) Application of homotopy analysis method to fourth order parabolic partial differential equations. Appl Appl Math 5:70-80

Misirli E, Gurefe Y (2011) Exp-function method for solving nonlinear evolution equations. Math Comput Appl 16:258-266

Momani S, Al-Khaled K (2005) Numerical solution for systems of fractional differential equations by the decomposition method. Appl Math Comput 162:1351-1365

Nassar HA, Abdel-Razek MA, Seddeek AK (2011) Expanding the tanh function method for solving nonlinear equations. Appl Math 2:1096-1104

Noor MA, Mohyud-Din ST, Waheed A (2008) Exp-function method for solving Kuramoto-Sivashinsky and Boussinesq equations. J Appl Math Comput. 29:1-13

Odibat Z, Momani S (2007) Numerical solution of Fokker-Planck equation with space-and time fractional derivatives. Phys Lett A 369:349-358

Ozis T, Koroglu CA (2008) Novel approach for solving the Fisher's equation using Exp-function method. Phys Lett A 372:3836-3840

Ray SS, Bera RK (2005) An approximate solution of a nonlinear fractional differential equation by Adomian's decomposition method. Appl Math Comput 167:561-571

Shawagfeh NT (2002) Analytical approximate solutions for nonlinear fractional differential equations. Appl Math Comput 31(2-3):517-529

Sirendaoreji (2004) New exact travelling wave solutions for the Kawahara and modified Kawahara equations. Chaos Solit Fract 19:147-150

Wang M, Li X, Zhang J (2008) The ( $\left.G^{\prime} / G\right)$-expansion method and travelling wave solutions of nonlinear evolution equations in mathematical physics. Phys Lett A 372:417-423

Wu HX, He JH (2007) Solitary solutions, periodic solutions and compacton like solutions using the exp-function method. Comput Math Appl 54:966-986

Yıldırım A, Kocak H (2009) Homotopy perturbation method for solving the space-time fractional advection-dispersion equation. Adv Water Resour 32:1711-1716

Yildirim A, Mohyud-Din ST, Sarıaydın S (2011)Numerical comparison for the solutions of an harmonic vibration of fractionally damped nano-sized oscillator. J King Saud Uni Sci. 23:205-209

Yusufoglu E (2008) New solitonary solutions for the MBBN equations using exp-function method. Phys Lett A 372:442-446

Zayed EME, Amer YA (2014) The first integral method and its application for.nding the exact solutions of nonlinear fractional partial differential equations (PDES) in the mathematical physics. Int J Phys Sci 9(8):174-183

Zayed EME, Zedan HA, Gepreel KA (2004) On the solitary wave solutions for nonlinear Hirota-Sasuma coupled KDV equations. Chaos Solit Fract 22:285-303

Zhang S (2007) Application of exp-function method to high-dimensional nonlinear evolution equation. Chaos Solit Fract 365:448-455

Zhou YB, Wang ML, Wang YM (2003) Periodic wave solutions to coupled KdV equations with variable coefficients. Phys Lett A 308:31-36

Zhu SD (2007) Exp-function method for the discrete mKdV lattice. Int J Nonlin Sci Num Simul 8:465-468 